\title{
Turning Towards Greater Equity and Access with Online Teacher Professional Development
}

\author{
Atom J. Lesiak', Joan C. Griswold ${ }^{1}$, and Helene Starks ${ }^{2}$ \\ ${ }^{1}$ Genome Sciences Education Outreach, Department of Genome Sciences and ${ }^{2}$ Department of Bioethics and Humanities, University of Washington, Seattle, WA \\ Keywords: Online teacher professional development, online PD, teacher in-service, distance learning, science education partnership award \\ Publication Date: August 30, 2021 \\ DOI: https://doi.org/10.15695/jstem/v4i3.05
}

\begin{abstract}
Access to professional development opportunities promotes equity for both teachers and students. We identified time, distance, and childcare needs as limiting factors for teachers seeking to attend our conference workshops and in-person professional development opportunities. Therefore, just months before the COVID-19 pandemic and the rapid shift to online learning, the Genome Sciences Education Outreach (GSEO) program transitioned to online professional development for the NIGMS sponsored SEPA grant Genes Environment and Me Network (GEMNet) program. Our program was well positioned to support teachers in our network by providing online PD that included technology support and rapid access to our online curriculum for use for distance learning. Here we share the process of creating online PD as well as converting our materials to the digital environment. Additionally, we provide survey data and lessons learned from eight online PD trainings from January 2020 to January 2021 for both Health/Family and Consumer Science Teachers (n=20) and Biology Teachers $(n=23)$. Notably, Teachers provided feedback affirming our initial reasons for providing online PD, and appreciated being supported at an overwhelmingly difficult time to be an educator. Our findings support the need for both online and in-person professional development opportunities for future programs.
\end{abstract}

\section{INTRODUCTION}

Teacher professional development (TPD) is essential to continually improve student outcomes by providing opportunities for teachers to improve their knowledge and practice (Avalos, 2011; Desimone, 2009; Garet et al., 2001). The need for remote online teacher professional development (oTPD) has risen dramatically during the COVID-19 pandemic as schools were rapidly forced to close, and physical distancing measures restricted in-person education for many students, teachers, and teacher educators (TE).

Despite the recent urgency, the need and call for oTPD is not new, as there has been strong advocacy for research and development regarding oTPD practices and pedagogical frameworks (Dede et al., 2009; Ganza, 2012; Ross, 2011). Proponents have highlighted that oTPD is comparable to in-person TPD and has the advantage of being an inexpensive way to increase teacher access to TPD opportunities (Brooks and Gibson, 2012; Darling-Hammond et al., 2017; Dede et al., 2009; Fishman et al., 2013). Many teacher educators were unprepared for the rapid shift away from in-per- son TPD, and the pandemic has highlighted the continuing need for better online engagement even after we return to in-person education and TPD (Hartshorne et al., 2020; Trust and Whalen, 2020). In September of 2020, Lay et al. (2020) compiled a comprehensive literature review of oTPD, highlighting how "the explosive growth of oTPD still outpaces rigorous empirical research." Equitable access to high quality PD opportunities is limited by each teacher's free time, financial considerations, geographic location, and child care responsibilities. While not the only solution to all of these limitations, oTPD can help ameliorate the travel burden on teachers in rural areas and can reach more teachers at minimal financial and environmental cost (Albers et al., 2015; Darling-Hammond et al., 2017; Lay et al., 2020; Powell and Bodur, 2019).

In this paper we describe our transition from in-person TPD to oTPD in the context of our NIH/NIGMS SEPA-funded program (GEMNet) that was in year three of data collection. The goal of the GEMNet Program is to devel- 


\section{Online Teacher Professional Development}

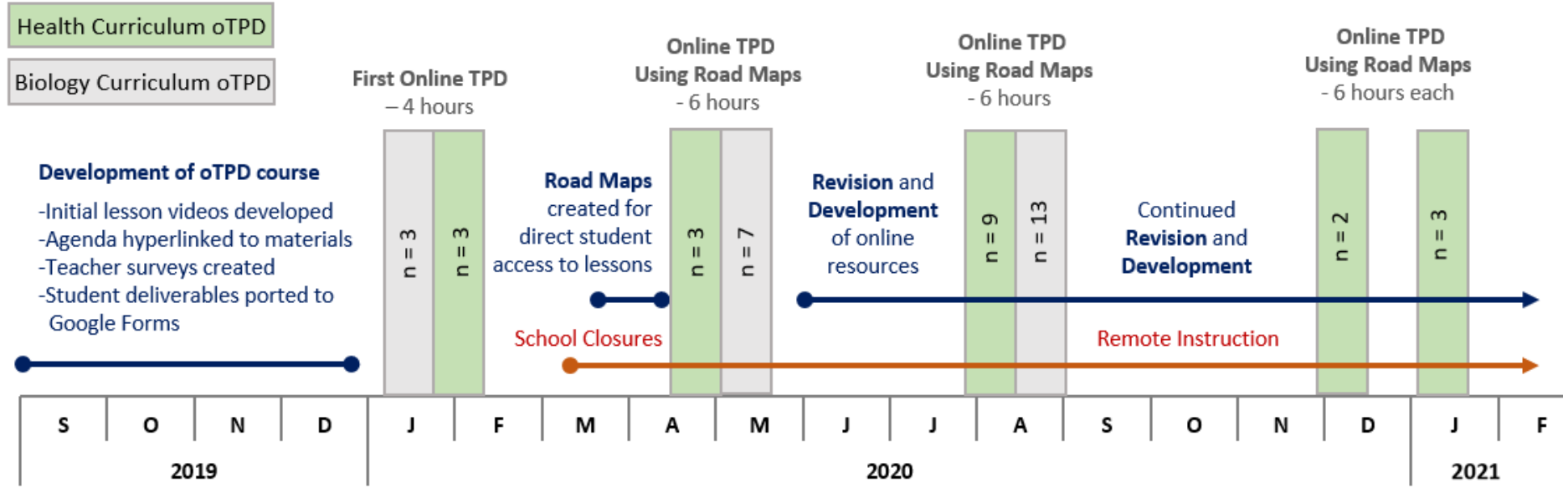

Figure 1. Graphical Representation of Online Teacher Professional Development.

op, implement, and evaluate two intersecting curricula on Type 2 diabetes for use by high school Health/Family and Consumer Science (Health/FACS) and Biology teachers, then measure the impact of the curricula for students receiving the curricula in either class or both Health/FACS and Biology classrooms. While recruiting teachers to join the GEMNet Program for PD, we noticed that many teachers had extremely limited time and resources to attend a partial, full, or multi-day session to learn our curriculum, and the GEMNet team began considering additional avenues of PD that would work well for teachers. In our initial grant proposal, we focused on traveling to conferences and other regional venues where teachers were gathering across our state to conduct PD, but coordination of scheduling day, place and time for multi-hour sessions highlighted the value-added in providing an online TPD opportunity. We had just completed our first oTPD session at the start of the pandemic and were therefore in position to adapt our curriculum more quickly to an online format and provide oTPD during the pandemic for over 43 teachers. Here we describe the processes of adapting our curriculum and TPD to an online format and share teacher feedback in hopes that others will gain insight from our experience.

\section{METHODS}

Creation and Description of Online Teacher PD. The GEMNet Diabetes curricula existed primarily as a multipage PDF document with accompanying slide-sets available to teachers on our website. During in-person TPD, we provided resources for teachers to experience the student lessons and accompanying hands-on activities for the five lessons in each of our curricula. In addition to delivering the necessary information for each lesson, these PD sessions provided community building opportunities among our teachers before, during, and after each session. Additionally, teachers would receive class sets of activity materials, and had the opportunity to engage in live conversations centered around the content of the lessons in the same way their students would ideally engage with the subject matter. In transitioning to an online TPD format we wanted to ensure that we balanced information delivery with group discussion opportunities for teachers to learn with and from other teachers. Before schools transitioned to remote education, we mailed class sets of activity materials to teachers. We plan to return to this practice when schools are back to in-person teaching.

Lead facilitators for the Health/FACS sessions and the Biology sessions were two of the GEMNet investigators. The oTPD sessions were conducted for Health/FACS and Biology teachers at different times between January 2020 through January 2021. Teachers were recruited to join us for online TPD using a mailing list compiled from all prior engagements and requests for follow up. As part of the GEMNet grant we offered teachers paid clock hours and compensation for their time $(\$ 25 /$ hour for first four, later increased to six hours total). Our first oTPD session in January 2020 consisted of two hours of synchronous online discussion and two hours of independent engagement with the curriculum. Thereafter, we increased the time offline to four hours because the teachers requested more time to prepare for discussion sessions (Figure 1). Teacher survey results after the first oTPD reflected their verbal desire for more independent time with the curriculum between online meetings.

\section{Objectives, Expectations, and Benefits from Online TPD Syllabus.}

\section{Course Objectives.}

As a result of our on-line professional development session, teachers will have:

- Ability to implement curriculum with a high level of fidelity 
- Increased health and science content knowledge

- Increased pedagogical knowledge and refined ideas about teaching, learning, and instructional strategies through deliberate reflection

- Higher self-confidence and motivation in curriculum use

- Deeper understanding of 3-D lesson design

- Gained knowledge of additional resources and lesson ideas

- Formed/engaged in a community of professional collaborators.

\section{Expectations:}

- Participants will complete all six hours of the course.

- If a teacher is not able to join a previously scheduled Zoom call, the teacher will be responsible for scheduling a make-up call with an instructor within one week of the missed call.

- Teachers will learn the curricula by processing the lessons as both students by completing the student sheets, and as teachers by answering reflection questions about the lessons.

- Teachers will complete and turn in all deliverables within three weeks of the start of the course.

\section{Benefits:}

- Teachers will learn new content and pedagogy that will be readily usable in the classroom

- Teachers will feel confident in teaching the GEMNet lessons

- Participants will be compensated $\$ 25 /$ hour, for a total of $\$ 150$ for the course

- Participants can receive six STEM Clock Hours

- Teachers will be eligible for additional compensation to participate in the GEMNet research study using the curriculum.

Online Lesson Materials. In our first online sessions, we merged the curriculum into a single PDF file with hyperlinks to each lesson and to important videos, online student worksheets, and teacher reflection surveys. For the oTPD, teachers completed a "deliverable" after reading each lesson, which was the equivalent to the student assessments that close out each lesson. In March 2020, we adapted our entire curriculum to online education, by re-writing each lesson for direct student access through the creation of online "student roadmaps." The roadmaps guided students through the lessons, step-by-step, with hyperlinks to videos, slidesets, activity instructions, and ending with links to complete student assessment forms. We used the same roadmaps to guide teachers through our oTPD, while encouraging them to access the original multi-page PDF format for additional teacher background information. Teachers then "learned by doing" the same activities as their students, ending each lesson with the same Student Sheet assessment followed by questions for teacher reflection. The GEMNet trainers monitored each teacher's completion of the assessments at the end of each lesson and identified aspects of the curriculum to clarify during online meeting time.

Online Meetings. Online meetings were carried out over three sessions, one week apart. Session 1 (45 minutes) introduced the goals and overview of online TPD with GEMNet. Session 2 (45 minutes) was organized around the discussion of the first three lessons of the curriculum. During Session 3 (30 minutes) we debriefed the last lessons of the curriculum and teachers completed a course evaluation and paperwork for continuing education clock hours and teacher stipends. Most important to the introductory session was to allow for technical troubleshooting, which was particularly helpful before teachers were familiar with technologies such as Zoom and Google Docs that were used extensively throughout the course. We provided teachers individualized assistance, as needed, outside these three sessions. We pre-recorded a short introductory video for teachers to watch before the first meeting to maximize time for teacher introductions and discussion. Over the course of the pandemic and the transition to online education, we found teachers valued this time to introduce themselves and share and brainstorm about the challenges they were facing. Teachers were expected to arrive at the second and third meetings with their completed activity sheets and ready to discuss the lessons covered between sessions. Teacher deliverables included completion of the online student sheets and activities for each lesson as well as a reflection on their experiences in the class. During live online meetings, teachers discussed each lesson, and GEMNet trainers helped clarify questions, address issues brought up through reflection, and share the experiences of teachers currently using the curriculum. In general, this approach led to efficient use of online screen time as the majority of online time was spent in group discussion of topics related to the lessons and implementation strategy.

Teacher Feedback Results. After oTPD, we received surveys back from 43 teachers over the course of eight trainings (Health/FACS n=20, Biology n=23) (Figure 2). Participants in oTPD trainings were high school teachers, primarily from rural, suburban, and urban Washington state, but included teachers from other states (Indiana, California, and Missouri). The survey was created to provide feedback to our organization and we did not collect demographics of teachers, school districts, or teaching history. The survey questions asked teachers to rate the utility of the oTPD, specific fea- 


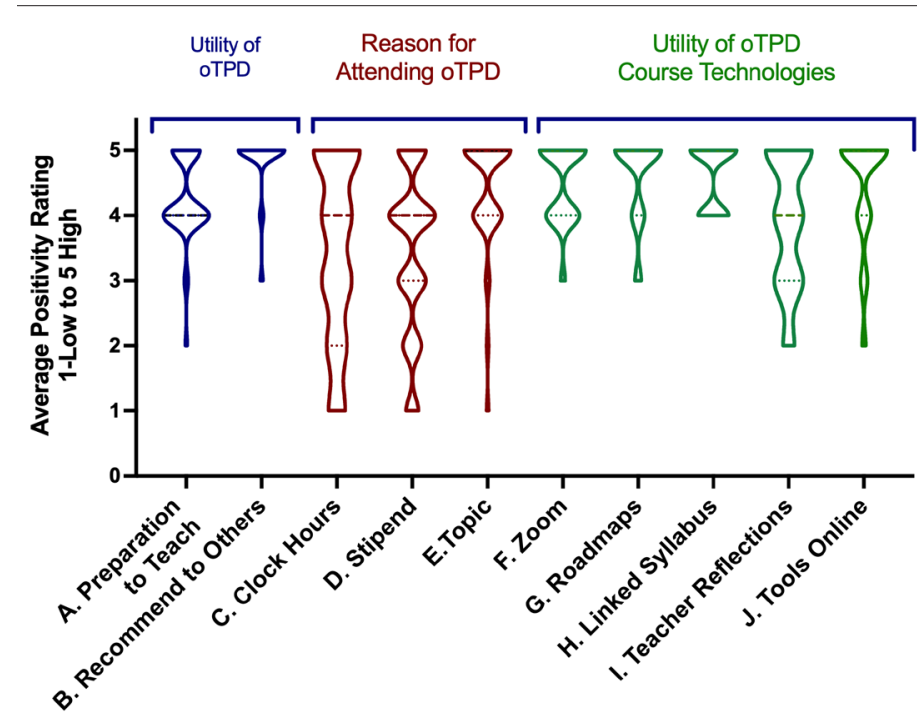

Figure 2. Combined Survey Results Regarding Exit Questions and Material Review. At the conclusion of each online TPD, teachers completed an exit survey. Teachers rated from $1=$ Negative/Unfavorable to $5=$ Positive/Favorable. A-B) Utility of oTPD. Data depicts Violin Plots demonstrating the distribution of teacher answers. Thick dotted line: Median, Thin dotted line: Quartiles. Survey prompts: A) Preparation to Teach the Curriculum. B) Likelihood to recommend to other teachers. C-E) Questions related to what inspired teachers to enroll in oTPD. C) Clock Hours. D) Stipend. E) Topic of Type 2 Diabetes. F-J) Teachers rated the utility of technologies used. F) Zoom Meetings and Discussions. G) Student and Teacher Roadmaps with hyperlinks. H) Course Syllabus with links to activities. I) Teacher reflections after completion of each lesson in the curricula.

J) Using online tools like Google Drive, Docs, Sites, Zoom, Typeform, etc. Teachers surveys varied slightly over the year. For an example Teacher Survey for Health/FACS can be found in Online Curriculum Resources.

tures of the course, and of their reasons for attending. All questions were rated using a 5 -point scale ( $1=$ negative/unfavorable; $5=$ positive/favorable). The overall responses were generally favorable (Figure 2). Of note, teacher survey data was never intended to be quantitative. The specific wording of questions asked in the teacher feedback survey changed slightly over the course of the year, and results reported in this study represent a compilation of survey results based on feedback topics from Jan. 2020 through Jan. 2021 (Figure 2). The use of a numerical rating scale (1-5) remained consistent throughout all surveys allowing for averaging of survey responses for each theme. Compiled survey results are qualitative findings intended to highlight the utility of our oTPD and should not be seen as quantitative research study results.

When it came to the utility of the oTPD sessions, most teachers felt like they left prepared to teach the curriculum and were likely to recommend oTPD with GEMNet to other teachers (Figure $2 \mathrm{~A}-\mathrm{B}$ ). Regarding their reasons for attending oTPD, teacher opinions were mixed regarding how much the offering of paid clock hours and payment for their time led to their participation, while interest in the topic of Type 2 Diabetes played a role for almost all teachers (Figure

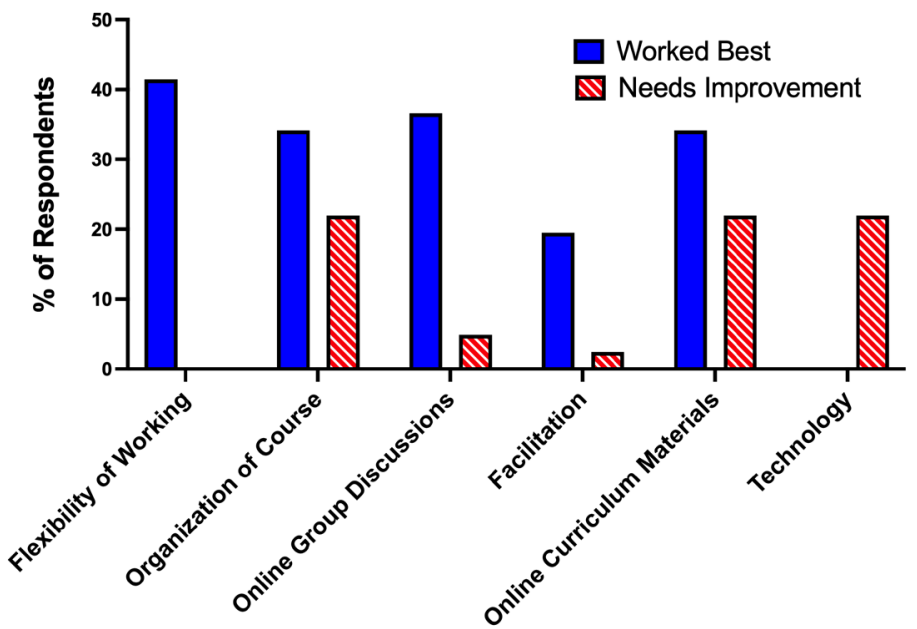

Figure 3. Categories of Teacher Comments. Teacher exit surveys allowed for written feedback on: BLUE - Which aspect of Online PD worked Best? and RED - Which aspects of Online PD need improvement. Comments from teacher surveys that provided feedback on these questions were categorized into specific categories. A) Flexibility of Working. B) Organization of Course. C) Online Group Discussions. D) Facilitation. E) Online Curriculum Materials. F) Technology. Graph depicts the percentage of survey respondents $(\mathrm{n}=43)$ that made mention to each category in their responses. For an example Teacher Survey for Health/FACS can be found in Online Curriculum Resources.

2 C-E). Regarding the utility of oTPD course technologies, teachers rated Zoom, Student Roadmaps, and hyperlinked syllabus highly. Teachers had mixed opinions about the utility of Teacher Reflections and the general online education resources (Tools Online) (Figure 2 F-J).

As part of the survey, teachers were given the opportunity to provide written feedback about the course (Figure 3 ). Teacher comments were compiled into specific categories for the prompts that asked about "What worked best?" and "What needs improvement?". When it came to positive comments, teachers rated highly the flexibility of working hours and the online groups discussions, and to a lesser extent facilitation by the GEMNet trainers. Teachers providing written feedback had mixed opinions related to the organization of the course and online materials. Usage of technology received no positive comments, and about $20 \%$ of the comments that we received noted technology as needing improvement. While we witnessed teachers become more competent with online tools, teacher's negative response to technology did not diminish over time.

When asked about whether the independent (flexible) engagement with the curriculum took the estimated amount of time, most teachers felt that they had adequate time, while a proportion of teachers would have appreciated more time. Health/FACS teachers were more likely than Biology teachers to feel as though they had enough time (Figure 4A). Teacher's overall rating of the online PD experience were generally high (Figure $4 \mathrm{~B}$ ). 


\section{DISCUSSION}

Online TPD in Future Programs. Ultimately, the transition to online TPD has been extremely successful for the GEMNet program. Teachers appreciated both the online-ready curriculum and online TPD, particularly during the stay-at-home orders and distance learning instruction. Accessibility, flexibility of material engagement combined with online socialization and discussion highlights the value of oTPD going forward (Powell and Bodur, 2019). Integration of online and in-person TPD will be a continuing facet of the GSEO/GEMNet programs.

Of note, our focus on equity and access for our teachers before the pandemic allowed for a relatively smooth transition to support teachers during a crisis. Many of the challenges towards equitable access to education have become increasingly clear during the pandemic, with childcare and elder care responsibilities rising to the forefront. Some teachers interested in in-person PD have barriers that prohibit their attendance, even if housing and travel costs are provided. Additionally, online spaces have emerged as a powerful space for community building, even if online meeting fatigue has become a challenge when this format becomes the only safe methodology for live discussions and socialization. During online meetings, especially early in the pandemic, facilitators noted that in our online spaces teachers expressed their appreciation for spaces to offer each other support and share their experiences. This value added should not be discounted, especially considering our ability to connect teachers from all over the country online.

Another major advantage to online TPD in addition to equity and access is that it is both cost effective and efficient. While the time cost to convert all of our materials for online education was high, both the online document management system (Google Drive) and online meeting platform (Zoom, paid for by University of Washington) were free. We had budgeted for extensive travel to national conferences and around the state of Washington for TPD, but ultimately spent a fraction of the total costs for online TPD. We were able to reach a significant number of teachers without leaving home and without the financial and environmental cost of travel for us or our participants.

Taking Hands-on Activities Online. One significant hindrance to adapting curriculum to the online environment was the inclusion of our "hands-on" activities. None of these activities were readily adaptable to the online environment, and it was a challenge to provide teachers with what they needed to still cover the lessons, particularly if their students had limited resources to recapitulate the activities at home. This is an on-going project with GEMNet as we produced online videos and a host of additional materials to support the hands-on lessons in an online format.

For our first session of online TPD, prior to the need for
A

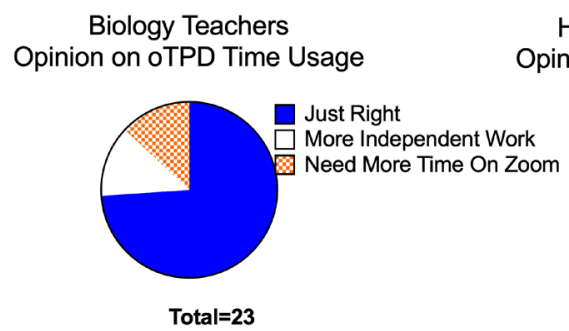

Health/FACS Teachers Opinion on OTPD Time Usage

B

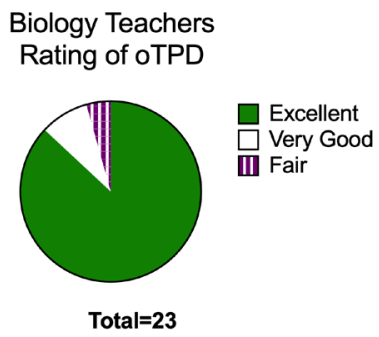

Health/FACS Teachers Rating of OTPD

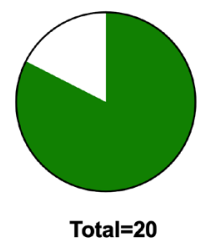

Figure 4. Teacher Opinions about Time Usage and Overall oTPD Review. Pie charts depicting teacher exit survey results for A) Completion of online TPD took the advertised amount of time. Options: Just Right, More than Expected, Less than Expected. B) Overall, how would you rate this Professional Development Training? Options: Excellent, Very Good, Good, Fair, Poor. For an example Teacher Survey for Health/FACS can be found in Online Curriculum Resources.

remote instruction, we developed and produced videos of students or staff completing the hands-on activities. Lesson 3 of the Biology curriculum is an example of one of these hands-on activities that went through multiple iterations because it uses a homeostasis model board (sheet) on which learners move board pieces (representing biomolecules) around to different organs in the body. After our first oTPD, before school closures, we followed up with teachers by mailing participating teachers class-sets of the activity materials. When schools transitioned to remote instruction, the videos of the model board were folded into the Student Roadmaps for direct student access. Over the course of the year, we developed four options for teachers in online PD to present the homeostasis model board to their students: showing their students how to create a board over Zoom, providing a manipulatable PowerPoint slide set to their students, showing a YouTube video of GEMNet staff using the model, or encouraging student to download a "DIY" model for at-home use. According to our YouTube statistics, most students accessed the video of the homeostasis model board in action with 1,153 views since April 2020. GSEO/ GEMNet is continuing to create engaging, on-line access for this important element of the curriculum. For an example of our online resources please see the Online Curriculum Resources in the Associated Content section below. For an example of the adapted hands-on activities see Lesson 3 of the Biology Curriculum. 
Technology, Internet Bandwidth, and Software Issues. The primary hurdle and major drawback to online TPD, and distance learning in general, is the navigation, compatibility, and quality of the technology. Internet connection, software compatibility, school district platform contracts, firewalls, blocked websites, and general computer issues will likely remain a challenge as technology improves and changes over time. The ability of both PD providers and teachers to adapt to technological advances will certainly be of the upmost importance as we work towards the future. Of note, the need to shift towards online engagement during the pandemic provided the impetus for many folks, including PD providers and teachers, to learn to navigate the technology needed to engage online. Time will tell how the rapid increase in live online engagement will impact education and society at large. We found that many of the early challenges just to get teachers connected with our online meeting platform dissipated dramatically as the year progressed. However, no amount of technology training can ameliorate the nationwide inequities regarding access to high-speed internet, and low bandwidth was an issue for both us as presenters as well as our teacher population.

Navigating the complex landscape of online document sharing was much more challenging. The biggest challenge for the Google Docs/Forms system we used was maintaining hyperlink integrity while making copies of all the Student Roadmaps and Forms for our teachers to deliver to their students. Each time a copy of the online curriculum was made, the hyperlinks between Roadmaps and Forms had to be updated and re-established before they were ready to send to students. Many of our teachers were already at capacity and we made significant efforts to create and maintain link integrity for many of our teachers. This would not have been sustainable if our teacher population was any larger. Eventually we created an in-depth guide for teachers to fix broken hyperlinks and manage folders containing all the documents they eventually sent out to their students. Unfortunately, each school district had different online education platforms and firewalls for various links and websites. The online converted curriculum didn't always synchronize nicely with the platforms of our teachers. This became a significant challenge as Student Roadmaps were converted into more engaging Google Sites. These Google Sites had to be abandoned when we found out that many school districts blocked access. The Google platform, including Google Sites, remained an option for sharing our course materials directly with teachers, if not students, with teachers using personal Gmail accounts when needed. For those considering online TPD we encourage finding more sustainable ways for teachers to make copies of online education materials if at all possible, and our group is currently looking for longterm solutions to streamline our current system. See Online Curriculum Resources listed in Associated Content.
Changing Role of the Instructor Online. When moving TPD and education online, the role of the instructor and the teacher changes, particularly using the Student Roadmap design. If learning can be primarily self-directed, are instructors and teachers still necessary? As instructors for online TPD we found that "flipping the classroom" by pre-recording lectures allowed a more effective use of time in Zoom meetings. Our role was transformed from instructor to discussion facilitator, guiding participants towards the learning objectives and away from unproductive tangents. Our topic of Type 2 Diabetes carries with it a significant social stigma and a complex interaction between genetic predisposition and life choices. With our curriculum, teacher facilitation of classroom discussions is imperative. Recurring themes in our oTPD sessions were about navigating classroom discussions, potentially offensive or triggering language, and ways to talk about food and diabetes without unintentionally promoting eating disorders. Facilitation of an asynchronous classroom discussion can be particularly challenging, as is hosting inclusive and engaging online discussions, especially when learners are attending the discussion from highly unpredictable environments.

\section{CONCLUSION}

Education and professional development online can be a highly effective and inclusive way to engage learners and it will likely be utilized increasingly over time. After the pandemic, we expect that many organizations will continue to use some form of online professional development. We hope that this programmatic description is of utility to others looking to provide online teacher professional development opportunities.

\section{ASSOCIATED CONTENT \\ Online Curriculum Resources.}

- GEMNet Website: https://gsoutreach.gs.washington. edu/programs/gemnet/

- Full GEMNet Type 2 Diabetes Online Curriculum (Biology and Health/FACS): https://sites.google.com/ uw.edu/gseo-online-lessons/home

- Example Online TPD Teacher Evaluation: https://forms. gle/4GvuAGP2RZsWPKGw7

- Example Hands-On Lesson

- Lesson 3 Biology Curriculum : https://sites.google.com/uw.edu/gemnet-bio-t2d-curriculum/ home\#h.7kf8v17atkz9

- Final Interactive Video Format via Typeform: https://atom814072.typeform.com/to/mmrZI17u 


\section{AUTHOR INFORMATION \\ Corresponding Author}

Atom J. Lesiak, Ph.D. Department of Genome Sciences, University of Washington. Seattle, WA, 98195. 970-2180193.alesiak@uw.edu

\section{Author Contributions}

The manuscript was written through contributions of all authors. All authors have given approval to the final version of the manuscript.

\section{ACKNOWLEDGMENTS}

The authors would like to thank Maureen Munn for her work creating Genome Sciences Education Outreach (GSEO) and creation of the GEMNet program. We want to thank all the hard work and dedication of the GEMNet Program teachers, and particularly acknowledge their fortitude during the 2020 pandemic.

\section{FUNDING SOURCES}

Funding for the Genes, the Environment, and Me: Health and STEM Network is provided by the National Institute of Health and National Institute of General Medicine Science Education Partnership Award (SEPA) 5R25GM129219-04.

\section{ABBREVIATIONS}

Health/FACS: Health/Family and Consumer Science; oTPD: Online Teacher Professional Development; TE: Teacher Educators; TPD: Teacher Professional Development

\section{REFERENCES}

Albers, P., Cho, A. R., Shin, J. H., Pang, M. E., Angay-Crowder, T., Odo, D. M., Jung, J. K., Pace, C. L., Sena, M., and Turnbull, S. (2015). Critical spaces for critical times: Global conversations in literacy research as an open professional development and practices resource. Global Education Review, 2(3).

Avalos, B. (2011). Teacher professional development in Teaching and Teacher Education over ten years. Teaching and Teacher Education, 27 (1), 10-20. https://doi.org/10.1016/j. tate.2010.08.007

Brooks, C., and Gibson, S. (2012). Professional learning in a digital age. Canadian Journal of Learning and Technology, 38(2).

Darling-Hammond, L., Hyer, M.E., and Gardner, M. (2017). Effective teacher professional development. Effective Teacher Professional Development (Research Brief), June.
Dede, C., Ketelhut, D. J., Whitehouse, P., Breit, L., and McCloskey, E. M. (2009). A research agenda for online teacher professional development. Journal of Teacher Education, 60(1), 8-19. https://doi.org/10.1177/0022487108327554

Desimone, L. M. (2009). Improving impact studies of teachers' professional development: Toward better conceptualizations and measures. Educational Researcher, 38(3). https:// doi.org/10.3102/0013189X08331140

Fishman, B., Konstantopoulos, S., Kubitskey, B. W., Vath, R., Park, G., Johnson, H., and Edelson, D. C. (2013). Comparing the impact of online and face-to-face professional development in the context of curriculum implementation. Journal of Teacher Education, 64(5). https://doi. org/10.1177/0022487113494413

Ganza, W. (2012). The impact of online professional development on online teaching in higher education. UNF Theses and Dissertations. http://digitalcommons.unf.edu/etd/345

Garet, M. S., Porter, A. C., Desimone, L., Birman, B. F., and Yoon, K. S. (2001). What makes professional development effective? Results from a national sample of teachers. American Educational Research Journal, 38(4), 915-945. https://doi. org/10.3102/00028312038004915

Hartshorne, R., Baumgartner, E., Kaplan-rakowski, R., Mouza, C., and Ferdig, R. (2020). Preservice and inservice professional development during the COVID-19 pandemic. Journal of Technology and Teacher Education, 28(2), 137-147.

John D. Ross. (2011). Online Professional Development: Design, Deliver, Succeed! Corwin Press.

Lay, C. D., Allman, B., Cutri, R. M., and Kimmons, R. (2020). Examining a decade of research in online teacher professional development. Frontiers in Education, 5. Frontiers Media S.A. https://doi.org/10.3389/feduc.2020.573129

Powell, C. G., and Bodur, Y. (2019). Teachers' perceptions of an online professional development experience: Implications for a design and implementation framework. Teaching and Teacher Education, 77, 19-30. https://doi.org/10.1016/j. tate.2018.09.004

Trust, T., and Whalen, J. (2020). Should teachers be trained in emergency remote teaching? lessons learned from the covid-19 pandemic. Journal of Technology and Teacher Education, 28 (2). 